\title{
Conservative subgroup separability for surfaces with boundary
}

\author{
MARK D BAKER \\ DARYL COOPER
}

If $F$ is a compact surface with boundary, then a finitely generated subgroup without peripheral elements of $G=\pi_{1}(F)$ can be separated from finitely many other elements of $G$ by a finite index subgroup of $G$ corresponding to a finite cover $\widetilde{F}$ with the same number of boundary components as $F$.

57M05; 20E26, 57M07, 57M10, 57N05

Suppose $F$ is a compact surface with nonempty boundary. A nontrivial element of $\pi_{1}(F)$ is peripheral if it is represented by a loop freely homotopic into $\partial F$. A covering space $p: \widetilde{F} \rightarrow F$ is called conservative if $F$ and $\widetilde{F}$ have the same number of boundary components: $|\partial F|=|\partial \widetilde{F}|$.

Theorem 0.1 (Main Theorem) Let $F$ be a compact, connected surface with $\partial F \neq \phi$ and $H \subset \pi_{1}(F)$ a finitely generated subgroup. Assume that no element of $H$ is peripheral. Given a (possibly empty) finite subset $B \subset \pi_{1}(F) \backslash H$, there exists a finite-sheeted cover $p: \widetilde{F} \rightarrow F$ such that:

(i) There is a compact, connected, $\pi_{1}$-injective subsurface $S \subset \tilde{F}$ such that $p_{*}\left(\pi_{1}(S)\right)=H$.

(ii) $\quad p_{*}\left(\pi_{1}(\widetilde{F})\right)$ contains no element of $B$.

(iii) $\tilde{F} \backslash S$ is connected and incl $l_{*} H_{1}(S) \rightarrow H_{1}(\widetilde{F})$ is injective.

(iv) The covering is conservative.

This theorem, for $F$ orientable and without (iii), is due to Masters and Zhang [5] and is a key ingredient in their proof that cusped hyperbolic 3-manifolds contain quasi-Fuchsian surface groups [4;5]. Without (iii) and (iv) the theorem is a special case of well-known theorems on subgroup separability of free groups (see Hall, Jr [1]) and surface groups (see Scott $[6 ; 7]$ ). For a discussion of subgroup separability and 3-manifolds, see Long and Reid [3].

The proof in [5] uses the folded graph techniques due to Stallings; see Kapovich and Myasnikov [2]. The shorter proof below uses cut and cross-join of surfaces. A cover is 
called good if properties (i)-(ii) hold and very good if (i)-(iii) hold. The idea is to start with a good cover and then pass to a second cover which is very good. Then cross-join operations (defined below) are used to reduce the number of boundary components of a very good cover until it is conservative.

\section{Constructing a very good cover}

We first explain a geometric condition on a cover of $F$ which ensures it is good, and then use Theorem 1.3 to construct a very good cover.

Choose a basepoint $x$ in the interior of $F$ and suppose $p: F_{H} \rightarrow F$ is the cover corresponding to $H$. There is a compact, connected, incompressible subsurface $S$ in the interior of $F_{H}$ which is a retract of $F_{H}$ and which contains a lift $\tilde{x}$ of $x$. Each element $g \in \pi_{1}(F, x)$ determines a unique lift $\tilde{x}(g) \in F_{H}$ of the basepoint $x$. The surface $S$ can be chosen large enough to contain $\{\tilde{x}(b): b \in B\}$. Then $\left.p\right|_{S}: S \rightarrow F$ is a local homeomorphism.

If $\pi: F^{\prime} \rightarrow F$ is any cover and there is a lift of $\left.p\right|_{S}$ to $\theta: S \rightarrow F^{\prime}$ (thus $\pi \circ \theta=\left.p\right|_{S}$ ) which is injective, we say $S$ lifts to an embedding in the cover $F^{\prime}$. The work of M Hall [1] and P Scott [6] shows there is a finite cover $F^{\prime} \rightarrow F$ such that $S$ lifts to an embedding in $F^{\prime}$.

Proposition 1.1 (Good cover) Under the hypotheses of the main theorem, if $\pi: F^{\prime} \rightarrow F$ is any cover and $S$ lifts to an embedding in $F^{\prime}$, then the cover is good.

Proof With the notation above, a based loop representing an element $b \in B$ lifts to a path in $F^{\prime}$ that starts at the basepoint $\tilde{x} \in L=\theta(S)$ but ends at some other point $\tilde{x}(b) \neq \tilde{x}$ in $L$.

Addendum 1.2 (Very good cover) There is a very good cover $\widetilde{F} \rightarrow F$ of finite degree with $|\partial \widetilde{F}|$ even.

Proof We start with a good cover $F^{\prime}$ of $F$ with finite degree and the subsurface $S \subset F^{\prime}$ described above and then construct a cover of $F^{\prime}$ with the required properties. Let $p: \widetilde{F} \rightarrow F^{\prime}$ be the regular cover given by the kernel of the map of $\pi_{1}\left(F^{\prime}\right)$ onto $H_{1}\left(F^{\prime}, S ; \mathbb{Z} / 2\right)$. There is a lift $\widetilde{S}$ of $S$ to this cover by construction. The conclusions follow from Theorem 1.3 below.

The following allows us to lift a $\pi_{1}$-injective subsurface to a regular cover where it is $H_{1}$-injective and nonseparating. 
Theorem 1.3 Suppose $F$ is a compact, connected surface, possibly with boundary, which contains a compact, connected subsurface $S \neq F$. Assume that $S \cap \partial F$ is a (possibly empty) union of components of $\partial F$ and no component of $\operatorname{cl}(F \backslash S)$ is a disc or a boundary parallel annulus. Let $p: \widetilde{F} \rightarrow F$ be the cover corresponding to the kernel of the natural homomorphism of $\pi_{1}(F)$ onto $G=H_{1}(F, S ; \mathbb{Z} / 2)$. If $\widetilde{S}_{0}$ is a connected component of $p^{-1}(S)$ then $X=\operatorname{cl}\left(\tilde{F} \backslash \tilde{S}_{0}\right)$ is connected and the map $i_{*}: H_{1}\left(\widetilde{S}_{0}\right) \rightarrow H_{1}(\widetilde{F})$ induced by inclusion is injective. Moreover $|\partial \widetilde{F}|$ is even.

Proof The hypotheses imply $G \neq 0$. Let $Y$ be a connected component of $X$. Then $\partial Y=(Y \cap \partial \widetilde{F}) \sqcup\left(Y \cap \widetilde{S}_{0}\right)$. We claim that $p(Y) \supset S$. Otherwise $\left.p\right|_{Y}: Y \rightarrow \operatorname{cl}(F \backslash S)$ is a covering map which is injective since $p \mid\left(Y \cap \widetilde{S}_{0}\right)$ is injective. Thus $Y$ is a lift of a component $Z$ of $\operatorname{cl}(F \backslash S)$.

If $Z \cap S$ is connected, then since $Z$ is not a disc or boundary parallel annulus, the image of $H_{1}(Z ; \mathbb{Z} / 2)$ in $G$ is not trivial. Thus $Z$ does not lift to the $G$-cover, a contradiction.

Hence $Z \cap S$ contains at least two distinct circle components $B_{1}, B_{2}$. There is a loop $\alpha=\beta \cdot \gamma \subset F$ which is the union of two arcs connecting $B_{1}$ and $B_{2}$ : one arc $\beta \subset Z$ and one arc $\gamma \subset S$. Since $\alpha$ has mod 2 algebraic intersection number 1 with the boundary component $B_{1}$ of $S$ it is a nonzero element of $G$. It follows that the lift $\tilde{\beta} \subset Y$ of $\beta$ has endpoints in different components of $p^{-1}(S)$, since otherwise $\alpha$ would lift to a loop. But $\partial \widetilde{\beta} \subset \partial Y \subset \partial \widetilde{S}_{0}$ which is a contradiction. Thus $p(Y) \supset S$.

It follows that $Y$ contains some component $\widetilde{S}_{1} \neq \widetilde{S}_{0}$ of $p^{-1}(S)$ in its interior. However the cover is regular so there is a covering transformation $\tau$ taking $\widetilde{S}_{0}$ to $\widetilde{S}_{1}$. Thus if $\widetilde{S}_{0}$ is not orientable then $Y$ is not orientable and if $\widetilde{S}_{0}$ contains a component of $\partial \widetilde{F}$ then so does $Y$.

Choose some Riemannian metric on $F$. This metric pulls back to one on $\widetilde{F}$ which is preserved by covering transformations. If $X$ is not connected, let $Y$ be a component of $X$ with smallest area.

As shown above, $Y$ contains an $\widetilde{S}_{1} \neq \widetilde{S}_{0}$ in its interior. The covering transformation $\tau$ taking $\widetilde{S}_{0}$ to $\widetilde{S}_{1}$ takes each component of $\widetilde{F} \backslash \widetilde{S}_{0}$ to a component of $\widetilde{F} \backslash \widetilde{S}_{1}$ with the same area. One of the components of $\widetilde{F} \backslash \widetilde{S}_{1}$ contains $\widetilde{S}_{0}$, so all the others must be strictly contained in $Y$, which contradicts that $Y$ has minimal area. Hence $X=Y$ is connected.

To show the injectivity of $i_{*}$, note the long exact homology sequence of the pair $\left(\widetilde{F}, \widetilde{S}_{0}\right)$ yields

$$
0 \longrightarrow H_{2}(\widetilde{F}) \stackrel{j_{*}}{\longrightarrow} H_{2}\left(\widetilde{F}, \widetilde{S}_{0}\right) \stackrel{\delta}{\longrightarrow} H_{1}\left(\tilde{S}_{0}\right) \stackrel{i_{*}}{\longrightarrow} H_{1}(\tilde{F}),
$$


So that we have the following equivalences: $\operatorname{ker} i_{*}=0$ if and only if Image $\delta=0$ if and only if $j_{*}$ is an isomorphism. By excision $H_{2}\left(\widetilde{F}, \widetilde{S}_{0}\right) \cong H_{2}\left(X, X \cap \widetilde{S}_{0}\right) \cong$ $H_{2}\left(X, \partial X \cap \partial \widetilde{S}_{0}\right)$.

Suppose $\partial F \neq \phi$. Then $X \cap \partial \widetilde{F} \neq \phi$, since otherwise $\partial \widetilde{F} \subset \widetilde{S}_{0}$, but we have shown $\tau\left(\widetilde{S}_{0}\right) \subset X$, which is a contradiction. Now $X \cap \partial \widetilde{F} \neq \phi$ implies $H_{2}\left(X, \partial X \cap \partial \widetilde{S}_{0}\right)=0$, so that Image $\delta=0$ hence $\operatorname{ker} i_{*}=0$.

The remaining case is $\partial F=\phi$. Here $X \cap \widetilde{S}_{0}=\partial \widetilde{S}_{0}=\partial X$. If $\widetilde{F}$ is orientable, then so is $X$, and it follows that $j_{*}$ is an isomorphism, hence ker $i_{*}=0$.

If $\tilde{F}$ is nonorientable, we claim $X$ must also be nonorientable; hence $H_{2}(X, \partial X)=0$ so that $0=$ Image $\delta=\operatorname{ker} i_{*}$.

Indeed, if $X$ is orientable then $\tilde{F}$ is orientable. This is because $\tau\left(\tilde{S}_{0}\right) \subset X$ so $\tau\left(\tilde{S}_{0}\right)$ orientable. This is a lift of $S$ so $S$ is orientable. Thus the homomorphism $\pi_{1}(F) \rightarrow \mathbb{Z}_{2}$ that sends a loop to 0 if and only if it is orientation preserving vanishes on $\pi_{1}(S)$ and so factors through $G$. It follows that every orientation reversing loop in $F$ has nonzero image in $G$ so $\widetilde{F}$ is orientable.

It remains to show $|\partial \widetilde{F}|$ is even. The action of $G$ on $\widetilde{F}$ is free. Since $\mathbb{Z}_{2}^{2}$ does not act freely on $S^{1}$ it follows that if $|\partial \widetilde{F}|$ is odd then $G \cong \mathbb{Z}_{2}$ and is generated by some component $C$ of $\partial F$. Let $Z$ be the component of $\operatorname{cl}(F \backslash S)$ that contains $C$. By excision $\mathbb{Z}_{2} \cong G \cong H_{1}(Z, Z \cap S)$. Since $Z$ is not a disc or an annulus with $C$ one of the boundary components the only other possibility is that $Z=\operatorname{cl}(F \backslash S)$ is a pair of pants with only one boundary component in $S$. But then $|\partial F|$ is even hence so is $|\partial \widetilde{F}|$.

The following is easily deduced from the proof of Theorem 1.3 and will be used in the next two sections of the paper.

Remark 1.4 If $F$ is a surface and $S \subset F$ is a subsurface and $X=\operatorname{cl}(F \backslash S)$ is connected and $X \cap \partial F \neq \phi$ then $i_{*}: H_{1}(S) \rightarrow H_{1}(F)$ is injective.

\section{Cross-joining covers}

Suppose $F$ is a surface and $\alpha_{1}$ and $\alpha_{2}$ are disjoint arcs properly embedded in $F$. Let $N\left(\alpha_{i}\right) \equiv \alpha_{i} \times[-1,1]$ be disjoint regular neighborhoods of the $\operatorname{arcs} \alpha_{i}$ in $F$ such that $\alpha_{i} \equiv \alpha_{i} \times 0$ and $N\left(\alpha_{i}\right) \cap \partial F=\left(\partial \alpha_{i}\right) \times[-1,1]$. The sets $\alpha_{i} \times(0, \pm 1] \subset F$ are called the \pm sides of $\alpha_{i}$. 
Given a homeomorphism $h: N\left(\alpha_{1}\right) \rightarrow N\left(\alpha_{2}\right)$ taking the + side of $\alpha_{1}$ to the + side of $\alpha_{2}$, the cross-join of $F$ along $\left(\alpha_{1}, \alpha_{2}\right)$ is the surface $K$ defined as follows. The surface $F^{-}=F \backslash\left(\alpha_{1} \cup \alpha_{2}\right)$ contains four subsurfaces $\alpha_{i} \times(0, \pm 1]$. Let $F^{\text {cut }}$ be the surface obtained by completing these subsurfaces to $\alpha_{i} \times[0, \pm 1]$. Thus $F^{\text {cut }}$ has two copies $\alpha_{i}^{+}, \alpha_{i}^{-}$of $\alpha_{i}$ in $\partial F^{\text {cut }}$ and identifying these copies suitably produces $F$. The surface $K$ is the quotient of $F^{\text {cut }}$ obtained by using $h$ to identify $\alpha_{1}^{-}$to $\alpha_{2}^{+}$and $\alpha_{1}^{+}$ to $\alpha_{2}^{-}$. Note that here we do not require $F$ to be connected, so that $\alpha$ and $\beta$ might be in different components of $F$.

There are two special cases of cross-join which will be used to change the number of boundary components of a surface:

Lemma 2.1 Suppose the compact surface $F$ contains two disjoint properly embedded arcs $\alpha$ and $\beta$. In addition suppose that

(1) either $F$ is connected and the endpoints of $\alpha, \beta$ lie on four distinct components of $\partial F$,

(2) or $F$ is the union of two connected components $A$ and $B$ and $\alpha \subset A$ has both endpoints on the same boundary component and $\beta \subset B$ has endpoints on distinct boundary components.

Then a surface $K$ obtained by cross-joining along these arcs has $|\partial K|=|\partial F|-2$. Furthermore $\chi(K)=\chi(F)$ and $K$ is connected.

Proof We verify that $K$ is connected. In the first case this follows since the arcs do not disconnect the boundary components on which they have endpoints; therefore $F \backslash(\alpha \cup \beta)$ is connected. In the second case it follows because $B \backslash \beta$ is connected, and every point in $K$ is connected to a point in this subset by an arc.

Suppose $p: \widetilde{F} \rightarrow F$ is a (possibly not connected) covering of surfaces and $\alpha$ is an arc properly embedded in $F$. Suppose $\widetilde{\alpha}_{1}$ and $\widetilde{\alpha}_{2}$ are two distinct lifts of $\alpha$ to $\widetilde{F}$; then they are disjoint. The map $p$ provides a homeomorphism between small regular neighborhoods of these two arcs. Using this to cross-join produces a surface $\widetilde{F}^{\prime}$ and since the identifications are compatible with $p$ there is a covering map $p^{\prime}: \widetilde{F}^{\prime} \rightarrow F$.

An important special case is when $\widetilde{F}$ is a $(d+1)$-fold cover which is the disjoint union of a 1 -fold cover $F_{1} \rightarrow F$ and some connected $d$-fold cover $F_{d} \rightarrow F$. Then cross-joining an arc in $F_{1}$ with one in $F_{d}$ produces a connected cover of degree $d+1$. To produce a new cover $F^{\prime}$ of $F$ by a cross-join along two arcs in some cover $\widetilde{F}$ requires the arcs to be disjoint from each other. If $S$ is embedded in $\widetilde{F}$ and these arcs 
are also disjoint from $S$, then $S$ lifts to an embedding in $F^{\prime}$, so the cover $F^{\prime}$ is good. We call the combination of these two properties the disjointness condition.

There is a metric condition, involving some arbitrary choice of Riemannian metric on $F$, that ensures the disjointness condition is satisfied and therefore that the new cover is good. The next lemma provides a uniform upper bound on the lengths of the arcs we will use to cross-join in any cover of $F$.

Lemma 2.2 (Short arcs) Suppose $F$ is a compact, connected surface with a Riemannian metric such that the diameter of $F$ is $\ell$. If $\widetilde{F}$ is a finite connected cover of $F$ then:

(1) If $A$ and $B$ are distinct components of $\partial F$ then there is an arc $\alpha$ in $F$ connecting them and length $(\alpha) \leq \ell$.

(2) If some component $A$ of $\partial F$ has (at least) two preimages in $\partial \widetilde{F}$ then there is an embedded arc $\alpha$ in $F$ of length at most $2 \ell$ which lifts to an arc with endpoints on distinct preimages of $A$.

Proof The first claim is obvious. For the second claim, since every point in $\widetilde{F}$ is within a distance at most $\ell$ of some point in $p^{-1}(A)$ and $\widetilde{F}$ is connected, some point in $\widetilde{F}$ is within a distance at most $\ell$ of points in two distinct components of $p^{-1}(A)$. This gives an $\operatorname{arc} \beta$ in $\widetilde{F}$ of length at most $2 \ell$ which connects two distinct components of $p^{-1}(A)$.

Let $\gamma:[0,2 R] \rightarrow \widetilde{F}$ be a shortest arc connecting two distinct components of $p^{-1}(A)$ and parametrized by arc length. Then $R \leq \ell$. To complete the proof we show that $\gamma$ projects to an embedded arc in $F$. Observe that

$$
d_{\widetilde{F}}\left(\gamma(t), p^{-1}(A)\right)=\min (t, 2 R-t)
$$

otherwise there is a shorter arc connecting two distinct components of $p^{-1}(A)$. It follows that

$$
d_{F}(p(\gamma(t)), A)=\min (t, 2 R-t)
$$

This means that the distance in $F$ of a point on $p \circ \gamma$ from $A$ is given by arc length along $p \circ \gamma$. It follows that $\alpha=p \circ \gamma$ is the required embedded arc.

An arc of length at most $2 \ell$ is called short. The next lemma provides a conservative cyclic cover with large diameter of a surface $F$. If a short arc in $F$ connects two distinct boundary components, then so does every covering translate of it. If $S$ lifts to the cover then there are many different translates of the short arc that are far from each other and far from the lift of $S$. In particular the disjointness condition is satisfied by suitable translates of a lifted short arc in this cover. 
Lemma 2.3 (Big covers) Suppose $F$ is a compact connected surface with $k \geq 2$ boundary components and which contains a compact, connected, incompressible subsurface $S \subset \operatorname{interior}(F)$ with $F \backslash S$ connected. Given $n>0$ there is a conservative finite cyclic cover $\widetilde{F} \rightarrow F$ of degree bigger than $n$ and a lift, $\widetilde{S}$, of $S$ to $\widetilde{F}$. Furthermore $\widetilde{F} \backslash \widetilde{S}$ is connected and the map $i_{*}: H_{1}(\widetilde{S}) \rightarrow H_{1}(\widetilde{F})$ induced by inclusion is injective.

Proof Let $Y$ be the surface obtained from $F \backslash \operatorname{interior}(S)$ by gluing a disc onto each component of $\partial S$. Then $Y$ is a connected surface with $k$ boundary components and there is a natural isomorphism of $H_{1}(F) / H_{1}(S)$ onto $H_{1}(Y)$. Choose a prime $p>\max (k, n)$. Because $Y$ is connected, there is an epimorphism from $H_{1}(Y)$ onto $\mathbb{Z} / p$ which sends one component of $\partial Y$ to $k-1$ and all the other $(k-1)$ components of $\partial Y$ to -1 . Now $(k-1)$ is coprime to $p$ because $2 \leq k<p$. Therefore this defines a conservative cyclic $p$-fold cover $\tilde{Y}$ of $Y$. It also determines a conservative cyclic $p$-fold cover $\tilde{F}$ of $F$ such that $S$ lifts to $\widetilde{S}$. Since $\tilde{Y}$ is connected, it follows that $X=\tilde{F} \backslash \widetilde{S}$ is connected. Also $X \cap \partial \widetilde{F}=\partial \widetilde{F}$ is not empty. Hence $i_{*}$ is injective by Remark 1.4.

\section{Proof of main theorem}

In this section all covers are of finite degree. Given a cover $p: \widetilde{F} \rightarrow F$, the excess number of boundary components $E(p)$ for this cover is defined as $E(p)=|\partial \widetilde{F}|-|\partial F|$. By Addendum 1.2 there is a very good cover $p: \widetilde{F} \rightarrow F$ with $|\partial \widetilde{F}|$ even. If $E(p)=0$ the theorem is proved; otherwise we construct another very good cover with smaller excess, and repeating this process a finite number of times yields a very good cover with zero excess.

These constructions use various very good covers of $F$. We will choose a lift of $S$ to each cover and identify this lift with $S$ and refer to the lift as $S$. This should not cause confusion.

We will also use the big cover Lemma 2.3 to replace a very good cover $\widetilde{F} \rightarrow F$ by another very good cover $F^{\prime} \rightarrow F$ with the same excess and very large diameter. This process is called taking a big cover. We will rename $F^{\prime}$ as $\widetilde{F}$.

Given $\tilde{F}$ very good, we will use (except in one case) one of the two cross-joins described in Lemma 2.1 to produce a new connected very good cover $F^{\prime}$ of $F$ with smaller excess. We first take a big cover so that there are lifts of a short arc that are far apart and disjoint from $S$ in $\widetilde{F}$. Then we change $\widetilde{F}$ with a cross-join to produce a good connected cover $F^{\prime}$, which has smaller excess by Lemma 2.1 . 
To verify $F^{\prime}$ is very good we check below that $F^{\prime} \backslash S$ is connected, then by Remark 1.4 this implies $\operatorname{incl}_{*} f: H_{1}(S) \rightarrow H_{1}\left(F^{\prime}\right)$ is injective, so $F^{\prime}$ is very good.

First observe that the cyclic cover produced by Lemma 2.3 leaves $\widetilde{F} \backslash S$ connected. Since the cross-join arcs are disjoint from $S$ they also determine a cross-join of $\tilde{F} \backslash S$. This cross-joined subsurface is $F^{\prime} \backslash S$ which is connected by Lemma 2.1 as required.

Case when $|\partial F|=1 \quad$ By Lemma 2.2 there is a properly embedded, short arc, $\alpha$, in $F$ which is covered by an $\operatorname{arc} \beta$ with endpoints on two distinct boundary circles of $\partial \widetilde{F}$. After taking a big cover we may assume the diameter of $\widetilde{F}$ is much larger than the length of $\alpha$ and the diameter of $S$; thus $\beta$ can be chosen to be disjoint from $S$ in $\widetilde{F}$. Cross-join $(\widetilde{F}, \beta)$ with $(F, \alpha)$ to obtain a cover $F^{\prime}$ with one fewer boundary circle than $\widetilde{F}$. There is a lift of $S$ to $F^{\prime}$ and $F^{\prime} \backslash S$ is connected. Repeat the process until we obtain a cover with only one boundary component. This completes the proof when $|\partial F|=1$.

Case when $|\partial F| \geq 2$ First we show how to make $E(p)$ even by performing a crossjoin if needed. This first step will increase the number of boundary components.

Suppose $E(p)$ is odd. By Addendum $1.2|\partial \widetilde{F}|$ is even, so $|\partial F|$ is odd. We can make $E(p)$ even by cross-joining $(\tilde{F}, \tilde{\alpha})$ and $(F, \alpha)$ to obtain a cover $p^{\prime}: F^{\prime} \rightarrow F$. To perform the cross-join, choose a short embedded arc $\alpha \subset F$ with endpoints on two distinct circles $C, C^{\prime}$ of $\partial F$ and a lift $\widetilde{\alpha} \subset \widetilde{F}$, with endpoints on two preimages $\widetilde{C}$, $\widetilde{C}^{\prime}$. After taking a big cover we can assume that $\tilde{\alpha}$ is disjoint from $S$ in $\widetilde{F}$. Then $F^{\prime}$ is the cross-join of $(F, \alpha)$ and $(\tilde{F}, \tilde{\alpha})$. The surface $S$ lifts to $F^{\prime}$ and $F^{\prime} \backslash S$ is connected by Lemma 2.1 .

Here is the outline of the rest of the proof. If $E(p) \neq 0$ then it is even. We proceed as follows using suitable cross-joins to construct new coverings. If there are two different components $C, C^{\prime} \subset \partial F$ which both have more than one preimage in $\partial \widetilde{F}$ then we find a short $\operatorname{arc} \alpha$ in $F$ connecting $C$ and $C^{\prime}$ and cross-join $\widetilde{F}$ to itself along two suitable lifts of $\alpha$ in $\tilde{F}$. This reduces the excess by 2 . After finitely many steps we obtain a cover so that at most one component $C \subset \partial F$ has more than one preimage. A single cyclic cross-join (defined below) is done simultaneously to reduce the excess to zero. Here are the details.

Suppose $A$ and $B$ are distinct circles in $\partial F$ which both have (at least) two distinct preimages $\widetilde{A}_{i}, \widetilde{B}_{i}$ for $i=1,2$ in $\partial \widetilde{F}$. Choose a short $\operatorname{arc} \gamma$ in $F$ with endpoints on $A$ and $B$. Let $\alpha_{i}$ be a lift of $\gamma$ with one endpoint on $\tilde{A}_{i}$ and $\beta_{i}$ a lift with an endpoint on $\widetilde{B}_{i}$. Inductively we assume that $\widetilde{F} \backslash S$ is connected. After taking a big cover we may assume that these arcs are all far apart and far from $S$. Thus there is a cover, obtained 
by cross-joining along any pair of distinct arcs chosen from this set of four, and $S$ lifts to this cover.

We claim that there is a pair of these arcs which have endpoints on four distinct boundary components of $\widetilde{F}$. It follows from Lemma 2.1 that cross-joining along this pair reduces the excess by 2 and $S$ lifts to the cover $F^{\prime}$ so produced. Furthermore, since $\widetilde{F} \backslash S$ is connected it follows that $F^{\prime} \backslash S$ is connected by Lemma 2.1 .

If $\alpha_{1}$ and $\alpha_{2}$ do not both have endpoints on the same lift $\widetilde{B}$ of $B$ the pair $\left(\alpha_{1}, \alpha_{2}\right)$ works. Similarly if $\beta_{1}$ and $\beta_{2}$ do not both have endpoints on the same lift $\tilde{A}$ of $A$ the pair $\left(\beta_{1}, \beta_{2}\right)$ works. The remaining case is (after relabeling) $\alpha_{1}$ and $\alpha_{2}$ both have endpoints on a component $\widetilde{B} \neq \widetilde{B}_{2}$ which covers $B$ and $\beta_{1}, \beta_{2}$ both have endpoints on some component $\tilde{A} \neq \widetilde{A}_{2}$ which covers $A$. Then $\alpha_{2}$ connects $A_{2}$ to $\widetilde{B} \neq \widetilde{B}_{2}$ and $\beta_{2}$ connects $B_{2}$ to $\tilde{A} \neq \tilde{A}_{2}$. Thus the pair $\left(\alpha_{2}, \beta_{2}\right)$ works.

Repeating this process a finite number of times reduces the excess by an even number until either $|\partial \widetilde{F}|=|\partial F|$ or else there is a unique component $C$ of $\partial F$ with more than one preimage. In the latter case the excess is even so there is an odd number of preimages $p^{-1}(C)=\left\{C_{0}, \ldots, C_{2 k}\right\}$.
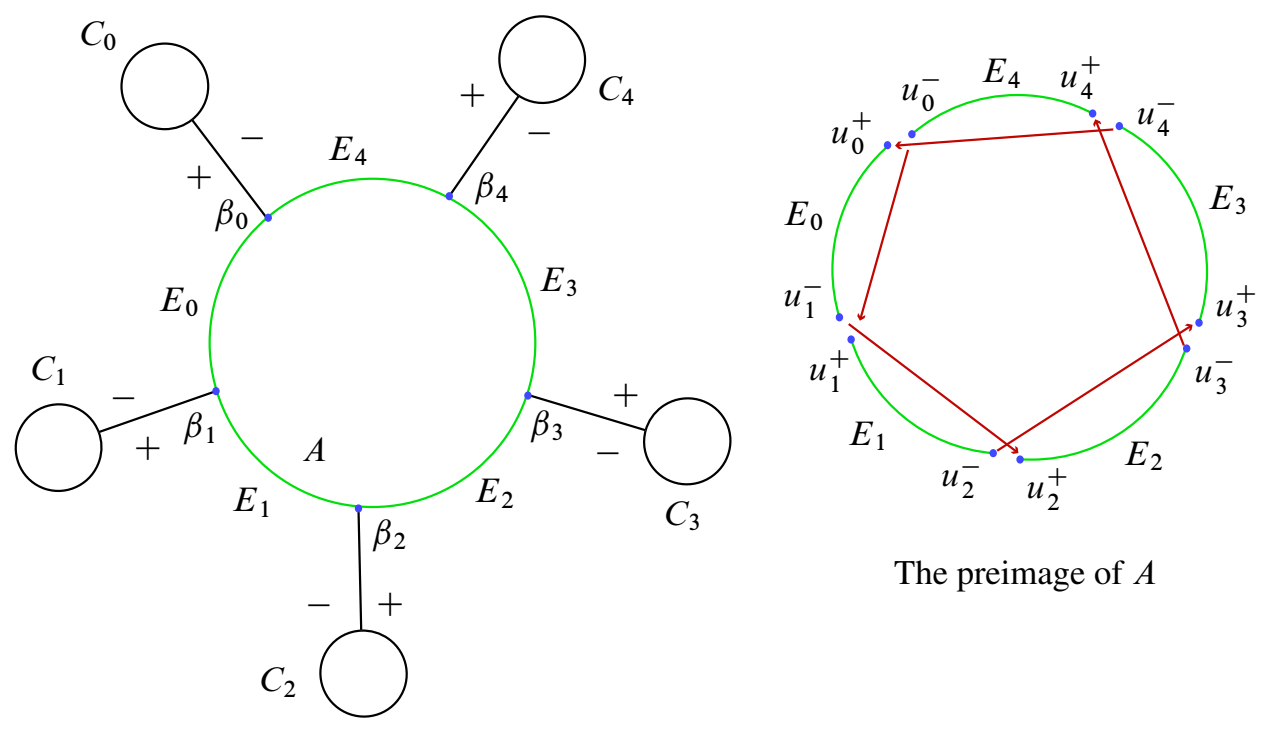

The preimage of $A$

Figure 1: Cyclic cross-joining, $2 k+1=5$ illustrated

Refer to Figure 1. Choose a component $A$ of $\partial \widetilde{F}$ that does not cover $C$. This is possible because $|\partial F| \geq 2$. Let $\beta$ be a short arc in $F$ with endpoints on $p(A)$ and $C$. For each $i$ there is a lift $\beta_{i}$ of $\beta$ with one endpoint on $C_{i}$ and the other on $A$. After 
taking a big cover we may assume all these lifts are far apart and far from $S$. Orient each arc $\beta_{i}$ so it points from $A$ to $C_{i}$ and call the left side + and the right side Now cross-join cyclically as follows. Cut $\widetilde{F}$ along the union of these arcs and join the - side of $\beta_{i}$ to the + side of $\beta_{i+1}$, with all integer subscripts taken mod $2 k+1$.

The resulting cover has a single preimage of $C$. Indeed, each $C_{i}$ has been cut at one point to give an interval $D_{i}=\left[t_{i}^{+}, t_{i}^{-}\right]$where the label $i$ denotes an endpoint of $\beta_{i}$ and $t_{i}^{ \pm}$is on the \pm side of $\beta_{i}$. These intervals are then glued by identifying $t_{i}^{-}$in $D_{i}$ to $t_{i+1}^{+}$in $D_{i+1}$. The result is obviously connected; it is a single circle.

To analyse the preimage of $p(A)$ the circle $A$ was cut at $2 k+1$ points to produce $2 k+1$ subarcs $E_{i}=\left[u_{i}^{+}, u_{i+1}^{-}\right]$where $u_{i}^{ \pm}$is on the \pm side of $\beta_{i}$. Then $E_{i}$ is glued to $E_{i+2}$ by identifying $u_{i+1}^{-}$with $u_{i+2}^{+}$(see figure 1 ). Since there are $2 k+1$ intervals and the $i$-th one is glued to the $(i+2)$-th one the result is connected because 2 is coprime to $2 k+1$. This gives the required conservative cover completing the proof of the main theorem.

Acknowledgements Cooper was supported in part by NSF grant number DMS0706887 and the CNRS. The authors thank the Institut Henri Poincaré for hospitality during the completion of this paper and the referee for helpful suggestions.

\section{References}

[1] M Hall, Jr, Coset representations in free groups, Trans. Amer. Math. Soc. 67 (1949) 421-432 MR0032642

[2] I Kapovich, A Myasnikov, Stallings foldings and subgroups of free groups, J. Algebra 248 (2002) 608-668 MR1882114

[3] D Long, A W Reid, Surface subgroups and subgroup separability in 3-manifold topology, 25 o Colóquio Brasileiro de Matemática 11, IMPA, Rio de Janeiro (2005) MR2164951

[4] J D Masters, X Zhang, Quasi-Fuchsian surfaces in hyperbolic link complements arXiv:0909.4501

[5] J D Masters, $\mathbf{X}$ Zhang, Closed quasi-Fuchsian surfaces in hyperbolic knot complements, Geom. Topol. 12 (2008) 2095-2171 MR2431017

[6] P Scott, Subgroups of surface groups are almost geometric, J. London Math. Soc. 17 (1978) 555-565 MR0494062

[7] P Scott, Correction to: "Subgroups of surface groups are almost geometric" [J. London Math. Soc. (2) 17 (1978), no. 3, 555-565], J. London Math. Soc. 32 (1985) 217-220 MR811778 
IRMAR, Université de Rennes 1

35042 Rennes Cedex, France

Department of Mathematics, University of California

Santa Barbara, CA 93106, USA

mark.baker@univ-rennes1.fr, cooper@math.ucsb.edu

http://www. math.ucsb.edu/ cooper/

Received: 19 April 2012 Revised: 19 September 2012 